\title{
汽车连接螺栓断裂的失效分析
}

\author{
贺 军
}

安徽中鼎精工技术有限公司，安徽 宣城 242000

[摘要]通过对汽车上连接螺栓在施工期间出现的断裂失效问题进行分析，采用外观检查、成分分析、金相检查等方法，判断 汽车连接螺栓断裂失效的原因, 最终研究结果显示, 造成断裂的原因是多源疲劳; 而结合汽车连接螺栓在使用期间的实际情 况, 认为汽车振动等造成螺栓松动, 造成应力集中部位的螺栓断裂。

[关键词]汽车连接; 螺栓断裂失效; 疲劳作用

DOI：10.33142/ec.v3i7.2341 中图分类号：U463 文献标识码：A

\section{Failure Analysis of Automobile Connecting Bolt Fracture}

HE Jun

Anhui Zhongding Precision Technology Co., Ltd., Xuancheng, Anhui, 242000, China

\begin{abstract}
Based on analysis of fracture failure of automobile connecting bolts during construction, the causes of fracture failure of automobile connecting bolts are determined by means of appearance inspection, composition analysis and metallographic examination. The final research results show that the cause of fracture is multi-source fatigue. Combined with actual situation of automobile connecting bolts during use, it is considered that automobile vibration causes the bolt causes bolt fracture in the stress concentration part.
\end{abstract}

Keywords: automobile connection; bolt fracture failure; fatigue effect

\section{引言}

螺旋的用钢要求具备较高的㓞性与强度, 被广泛应用在汽车连接位置, 成为保证汽车正常运行的关键因素。但是 实际情况证明, 汽车连接螺栓断裂失效的情况较为常见, 该现象不仅会影响汽车的性能, 还会增加汽车安全行驶风险, 值得关注。

\section{1 案例简介}

某汽车使用了高强度的连接螺栓, 该螺栓的使用级别为 12.9 级, 且主要原材料为 $35 \mathrm{CrMoA}$ 钢, 在表面镀锌。该螺 栓在车辆运行期间出现了断裂, 遂进行检查。从外观观察来看, 螺栓断裂位置位于第 2-3 扣的螺纹处, 距离螺栓六方 头断面的距离约为 $40 \mathrm{~mm}$ 。初步分析造成变形的主要原因是销子反复挤压, 造成螺栓的变形、磨损。

\section{2 断裂螺栓的试验与研究}

\section{1 外观检查}

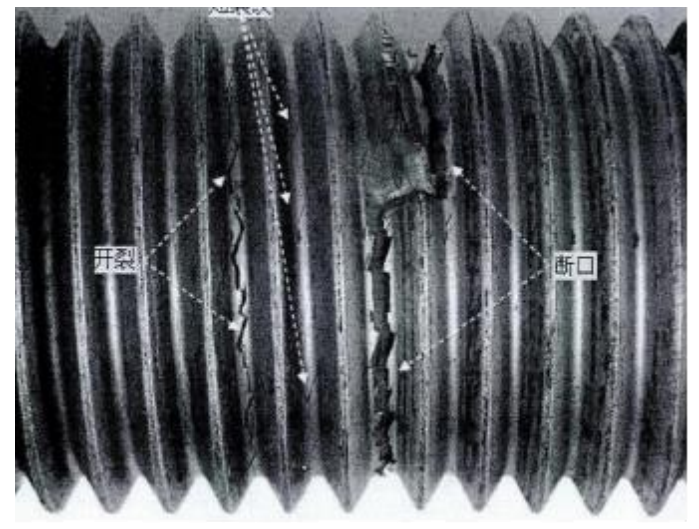

图 1 螺栓的损伤情况 
在接触螺栓之后, 发现断裂螺栓的螺纹顶部有严重的镀锌层磨损脱落的情况, 认为该位置出现了严重的损伤。在 对损伤部位, 沿着直径轴向的方向做切割, 获得金相试样之后, 于显微镜下观察, 发现该螺栓的第 3-10 根螺纹的根部 位置有明显的裂纹情况; 螺纹累及牙顶, 导致螺栓牙顶位置出现了严重的破坏, 部分严重位置有明显的翻边 (见图 1)。 之后结合螺栓的使用情况, 认为造成这一现象的原因, 是在螺栓松动之后, 与发动机连接的零部件与螺栓之间出现了 反运动, 之后随着车辆的行驶, 反运动越来越明显, 在反复的撞击下造成螺纹出现了损坏。

从螺栓的整体结构来看, 螺栓端口位置相对平坦, 两端的接口可良好配合, 且无明显的塑性变形; 进一步观察, 发现端口处的颜色浅, 且平整; 中间位置的颜色深, 且相对粗粘; 在断口位置可发现疲劳弧线, 弧线从两端逐渐向中 心位置发展, 并形成了狭窄的撕裂脊线。造成这一现象的原因考虑可能为: 两侧疲劳的裂纹在外力作用的影响下, 同 时向内扩展并最终相遇, 由此形成了这种特殊的结构, 且这种裂纹也满足双向疲劳断裂的特征, 很少发现瞬断区 (本 次研究发现, 进口的瞬断区主要集中在断口边缘位置)。

\section{2 成分分析}

通过对该螺栓做化学成分检验, 具体的检测结果如表 1 所示, 而最终的成分分析结果也证明, 该配比满足相关标 准中有关 $35 \mathrm{CrMoA}$ 的技术要求, 证明该螺栓的主要原材料配比不存在质量问题。

表 1 成分分析结果

\begin{tabular}{c|c|c|c|c}
\hline 检测项目 & 钼 & 硅 & 锰 & 铬 \\
\hline 标准值 & $0.15-0.25$ & $0.17-0.37$ & $0.40-0.70$ & $0.80-1.10$ \\
\hline 实测值 & $0.17-0.18$ & $0.24-0.28$ & $0.52-0.54$ & $1.03-1.05$ \\
\hline
\end{tabular}

\section{3 金相检验}

针对断裂螺纹螺栓, 在沿着纵轴向方向切开后, 做金相检验。检查结果显示, 基体的纤维组织主要包括少量的铁 素体与回火索氏体, 螺纹的根部未发现脱碳现象。且最终的显微梯度检查结果发现, 螺纹部分无明显硬化现象。

而结合该螺栓在汽车连接件的实际情况进行考察, 发现螺栓有部分螺纹并没有被旋入零件中。在正常的条件下, 螺纹与螺栓之间保持了良好的预紧力, 能够使螺栓与零件之间形成一个整体, 车辆行驶期间所产生的振动能量将会被 其他零件上的柔性橡胶连接体等吸收; 但是如果发生了螺栓预紧力不足, 或者在外界剧烈振动的情况下, 都会导致螺 旋出现松动情况, 螺栓与零件之间会产生不同程度的相对运动, 这个相对运动将会使与发动机连接的零件, 将发动机 所产生的振动力传输到其他位置, 而靠近零件位置的螺纹所承受的弯矩最大, 可能成为断裂源, 这也螺栓中螺纹根部 出现疲劳裂纹的重要原因。

\section{4 断口分析}

在对断裂的螺栓做充分清洗后, 放置在显微镜观察, 采用低倍形貌观察后, 可以发现断口靠近边缘位置细淢而平 坦, 此位置为裂纹源区; 之后逐渐向中间位置发展, 逐渐变得粗粘, 此位置为扩展区。在对瘦劳源区 (见图 2) 做观察 后, 发现裂纹源自于螺纹根部较深位置, 可发现明显的疲劳擦伤, 而裂纹有明显的台阶状特征, 证明属于多源断裂情 况。在对疲劳裂纹扩展区进行观察后, 发现二次裂纹的数量要显著高于裂纹岭, 且断口位置具有间距小、疲劳条带短 等问题, 此现象证明螺栓的使用期间出现了较高的疲劳损伤。

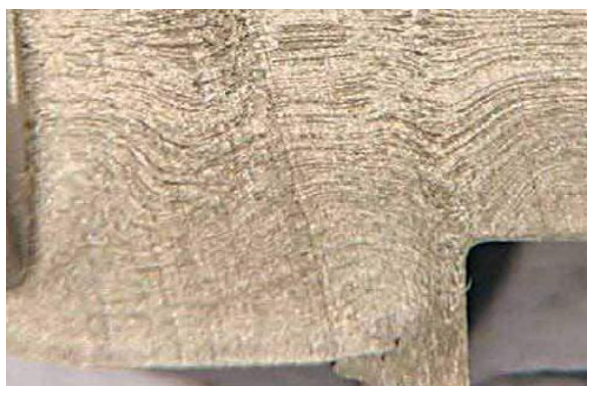

图 2 疲劳源区的显微镜观察结果 
在观察断裂的韧窝特征后, 发现韧窝小, 并表现出等轴状的特征, 证明该区域进出线在断口边缘的范围小, 这一 观察结果显示, 在最后断裂情况下螺栓所承受的应力小。这是因为在螺纹扩张的情况下, 施加在螺栓上的径向力因为 开裂的影响, 使位移量释放; 而汽车所产生的振动幅度基本是相同的, 这种特殊的施加外力的情况会导致裂纹长期处 于不断扩大的过程中, 相应的有效承载面积也在不断减小, 所承担的应力不断扩大; 在螺栓断裂的一瞬间, 出现了一 次性瞬断的情况，这种情况也与本文的显微镜观察结果是相同的。

\section{3 结果分析}

\section{1 本文的研究结果}

根据上述的观察结果, 最终本文研究证明, 造成该螺栓失效的主要原因是弯曲疲劳断裂; 根据金相分析结果, 在 螺纹部分未发现明显的脱碳情况, 且各项微组织基本正常, 证明该零部件在冶炼期间不存在质量缺陷。各项成分调查 结果显示, 从表 1 的相关数据, 可发现该零部件的各项主要原材料成分均满足预先的设计要求, 且螺纹部分与基体的 硬度相同。因此在进行上述诸多分析后, 最终本文认为, 造成汽车连接螺栓失效断裂的原因可能是预紧力不足, 或因 为发动机在运行期间所产生的振动而导致螺栓在长期的受力作用下发生了松动。根据前文的研究可认为, 零部件在与 螺栓产生相对运动之后, 发动机在运行期间, 会通过零部件将自身的振动力传递给螺栓, 导致螺栓承受了较高的交变 应力, 而靠近零件的螺纹, 所承受的弯矩力较大, 可能成为断裂源。

同时, 考虑到螺栓在加工生产过程中, 生产质量必然会受到工艺等因素的限制, 大部分螺纹根部的圆周角曲率半 径要小于预期设计值, 且光洁度难以达到预期标准, 因此有可能出现应力相对集中的情况, 造成应力集中的系数持续 增加。

所以在进行上述分析后, 认为该螺栓首选是沿着应力集中的位置, 部分螺纹根部发生了断裂情况, 之后在相邻的 多个螺纹根部也开始出现疲劳裂纹情况, 最终造成螺栓失效。

\section{2 预防措施}

汽车螺栓失效的问题难以从根本上杜绝, 所以为了能够保证行车安全, 在未来的工作中应该做到以下几点问题, 争 取能够将螺栓断裂失效的风险降到最低, 保证行车安全。(1) 所选购的螺栓必须要经过国家专业的质量检验, 严禁使用 存在质量缺陷的螺栓, 从源头上降低螺栓断裂的风险。(2) 在安装螺栓期间, 应保证螺栓的预紧力良好, 按照加工标准 将螺栓拧紧、拧牢, 并做好质量检测, 避免螺栓的预应力不足而出现质量问题。(3) 定期对螺栓的状态进行检测, 使用 无损检测技术, 判断螺栓的内部状态变化, 在发现螺纹根部发生断裂情况后, 应及时检查断裂的原因并更换零部件; 针 对严重破坏的螺栓, 要从多个角度分析破坏的原因, 并采取预防处置措施, 使螺栓失效问题可得到有效处置。

\section{4 结束语}

本次研究中, 所选择的案例螺栓失效原因为多元疲劳断裂, 而造成断裂的原因可能是预紧力不符合要求, 或者发 动机/汽车的振动、颠䈱而导致螺栓松动, 导致螺栓持续受到交变应力的影响, 这是造成损伤的重要原因。除此之外, 加工质量问题也对螺栓性能产生影响, 导致螺栓的断裂失效

[参考文献]

[1]苏倩, 胡春燕.汽车下摆臂与车架连接螺栓断裂分析 [J]. 失效分析与预防, 2019, 14(06): 425-428.

[2] 孔德群,周建,杨利.汽车喷油嘴螺栓断裂的失效分析 [J].金属加工 (热加工), 2019 (10): 77-79.

作者简介: 贺军 (1985. 8-), 男, 佳木斯大学, 生物医学工程, 安徽中鼎精工技术有限公司, 检测中心主任, 工程师 技术资格。 